\title{
Cerebral Small Vessel Disease and Arterial Stiffness: Tsunami Effect in the Brain?
}

\author{
Naoki Saji Kenji Toba Takashi Sakurai \\ Center for Comprehensive Care and Research on Memory Disorders, \\ National Center for Geriatrics and Gerontology, Aichi, Japan
}

\section{Key Words}

Ankle-brachial index - Arterial stiffness - Cerebral small vessel disease - Cognitive impairment . Pulse wave velocity

\begin{abstract}
Background: Cerebral small vessel diseases, including silent lacunar infarcts, white matter hyperintensities, and microbleeds, pose a risk for cerebrovascular disease, cognitive impairment, and the geriatric syndrome via effects on arterial stiffness. However, the vascular, physiological, and metabolic roles of arterial stiffness in cerebral small vessel diseases remain unclear. Summary: Arterial stiffness can be assessed using various indicators such as the ankle-brachial index, pulse wave velocity, cardio-ankle vascular index, and augmentation index. Arterial stiffness is independently associated with all components of cerebral small vessel disease including silent lacunar infarcts, white matter hyperintensities, and microbleeds, although there are some methodological differences between the various surrogate markers. Evidence of arterial stiffness indicates microvessel arteriosclerosis presenting with vascular endothelial dysfunction. Further, vascular narrowing due to atherosclerosis and vascular stiffness due to lipohyalinosis can accelerate the pulse waves. This hemodynamic stress, pulsatile pressure, or blood pressure variability can cause a 'tsunami effect' towards the cerebral parenchyma and lead to cerebral small vessel disease. Previous studies have shown that silent lacunar infarcts and white matter hyperintensities are strongly associated with arterial stiffness. However, the association between microbleeds and arterial stiffness remains controversial, as there are two vessel mechanisms related to microbleeds: cerebral amyloid angiopathy and hypertensive small vessel disease. Key Messages: Cerebral small vessel disease with as-
\end{abstract}


Saji et al.: Cerebral Small Vessel Disease and Arterial Stiffness: Tsunami Effect in the Brain?

sociated arterial stiffness is a risk factor for silent cerebral lesions, stroke, and cognitive impairment. Improvement of the living environment, management of risk factors, and innovation and development of novel drugs that improve arterial stiffness may suppress the progression of cerebral small vessel disease, and may reduce the risk for stroke and dementia.

(C) 2016 S. Karger AG, Base

\section{Introduction}

Cerebral small vessel disease (SVD) is the most common vascular cause of dementia, a major contributor to mixed dementia, and the cause of approximately one fifth of all strokes worldwide [1,2]. Recently, the neuroimaging features of SVD on magnetic resonance imaging (MRI) have been reclassified into five categories: (1) recent small subcortical infarcts (formerly categorized as acute lacunar stroke); (2) white matter hyperintensity (WMH); (3) lacunae [formerly defined as silent lacunar infarcts (SLI)]; (4) microbleeds (MB), and (5) perivascular space [1].

Cerebral SVD is of clinical importance as a risk factor for cerebrovascular diseases and cognitive impairment $[1,2]$. There is also widespread acceptance of the existence of vascular cognitive impairment. Additionally, the continuity between cerebrovascular diseases and cognitive impairment is of increasing interest. Cerebral SVD poses a risk for both cerebrovascular disease and cognitive impairment via effects on arterial stiffness, presenting as microvessel arteriosclerosis with vascular endothelial dysfunction [3]. Recent studies also suggest an association between arterial stiffness and the geriatric syndrome [4, 5].

Stroke, dementia, and the geriatric syndrome are important in our aging society, as such diseases increase caregiver burden [6]. The increased number and proportion of older adults presenting with these diseases in the Japanese population and worldwide is heightening the financial pressures on health care systems. Here, we review the characteristics of cerebral SVD from the viewpoint of silent cerebral lesions, stroke, and dementia, with a particular focus on the association with arterial stiffness.

\section{Arterial Stiffness}

\section{Outline}

Arterial stiffness is a known predictor of mortality, cardiovascular diseases, and cerebrovascular diseases [7-9]. Recently, numerous studies have also shown novel associations between cognitive impairment and arterial stiffness [10-15]. Methods for assessing arterial stiffness are noninvasive and allow simple assessment and data evaluation. Surrogate markers to assess arterial stiffness include the ankle-brachial index (ABI), pulse wave velocity (PWV; e.g., aortic, brachial-ankle, and carotid-femoral PWV), cardio-ankle vascular index (CAVI), and augmentation index. A decreased ABI indicates atherosclerotic changes in large arteries [9]. By contrast, an increased ABI indicates progression of arterial stiffness [13]. Therefore, the $\mathrm{ABI}$ is considered a component of arterial stiffness. Although there are some methodological differences between these markers, their overall utility is well established.

\section{Measurement}

Patients were placed in the supine position and rested for $5 \mathrm{~min}$ before the measurements of arterial stiffness commenced. Electrocardiographic electrodes were placed on both wrists, cuffs were wrapped around both ankles and the brachial regions of both forearms, and the heart sounds were monitored. Firstly, ABI was calculated by the ratio of the systolic blood 
Saji et al.: Cerebral Small Vessel Disease and Arterial Stiffness: Tsunami Effect in the Brain?

pressure at the ankle to the systolic blood pressure at the arm. Secondly, brachial-ankle PWV was automatically calculated according to the height of the patients, the distance between the brachium and the ankle, and the time intervals between the wave at the right brachium and those at both ankles [16]. Thirdly, CAVI was calculated independent of blood pressure using the stiffness parameter $\beta$ in the Bramwell-Hill equation [17]. The stiffness parameter $\beta$ indicates patient-specific, blood pressure-independent vascular stiffness as measured by arterial ultrasound. The detailed formula of CAVI is shown elsewhere $[17,18]$.

\section{Cerebral SVD}

\section{Outline}

Cerebral SVD such as WMH, SLI, and MB are considered to involve silent cerebral changes. Firstly, WMH characterized by bilateral, mostly symmetrical hyperintensities on T2-weighted MRI are common in older individuals. WMH is strongly associated with cerebrovascular disease, vascular risk factors, and covert neurological and cognitive symptoms and physical difficulties such as gait disturbance [1]. Secondly, SLI are frequently seen on imaging in older patients with no symptoms and are associated with an increased risk of stroke, gait impairment, and dementia [1]. Thirdly, MB are small hypointense lesions that are visible on T2*-weighted gradient-recalled echo or susceptibility-weighted sequences, and are most commonly located in the cortico-subcortical junction and deep gray or white matter in the cerebral hemispheres, brainstem, and cerebellum [1]. MB are associated with cognitive impairment such as Alzheimer's disease.

\section{Assessment and Clinical Meaning}

We defined WMH as an irregular periventricular hyperintensity (Fazekas grade $\geq 3$ ) and/ or early confluent or confluent separate deep white matter hyperintense lesions (Fazekas grade $\geq 2$ ) on FLAIR images [19]. We defined SLI as a focal lesion of $\geq 3 \mathrm{~mm}$ in diameter, with hyperintensity on T2-weighted MRI and hypointensity on FLAIR [20]. We defined MB as a focal area of signal loss in the brain parenchyma $<5 \mathrm{~mm}$ in size on T2* scans [21].

Cerebral SVD is the most common vascular cause of stroke and dementia, and is a major contributor to mixed dementia [1]. In fact, these lesions are not 'silent', but rather they slowly increase the risk of stroke and dementia in the brain. For example, as the numbers of SLI and MB increase, the risk of cerebrovascular diseases and cognitive impairment increases. Likewise, as the area of WMH enlarges, the risks of disease increase. Age and hypertension are well-known risk factors for cerebral SVD [22]. Pulse pressure and blood pressure variability may also contribute to the risk of cerebral SVD and cardiovascular and cerebrovascular diseases $[23,24]$.

\section{Association with Arterial Stiffness}

Arterial stiffness is independently associated with cerebral SVD including WMH [19], SLI $[20,25]$, and MB [26]. The mechanism of cerebral SVD is considered to involve vascular endothelial dysfunction, which may be associated with arterial stiffness [27]. Further, blood-brain barrier failure or progression of arteriosclerotic changes may lead to cerebral parenchymal damage [3]. As there are vessel similarities between the kidney and the brain, the presence of chronic kidney disease may also be associated with cerebral SVD based on similar mechanisms $[21,28]$.

Taken together, there is a robust association between cerebral SVD and arterial stiffness, although there are some methodological differences between the surrogate markers of arterial stiffness. Both WMH and SLI are independently associated with increased PWV [25]. 
Saji et al.: Cerebral Small Vessel Disease and Arterial Stiffness: Tsunami Effect in the Brain?

By contrast, the independent association between CAVI and WMH remains controversial, although CAVI is independently associated with SLI [20]. This discrepancy suggests that (1) microvessel arteriosclerosis such as lipohyalinosis is a major cause of SLI and (2) vessel stiffness, as well as other factors such as pulsatile pressure, may affect the etiology of WMH. The associations between PWV, CAVI, and MB are also controversial, which may be related to the different etiology of MB: the differences between deep and/or infratentorial MB and lobar MB. Deep MB located in the basal ganglia are mainly caused by hypertensive SVD, while lobar MB are caused by cerebral amyloid angiopathy [21]. Thus, both positive and negative associations between MB and arterial stiffness are expected, and are dependent on the differences in participating subjects.

\section{Stroke}

Stroke is a major cause of long-term care in Japan [6]. Lacunar infarcts are a common subtype of brain infarction [22]. Acute lacunar infarcts are associated with WMH [22] and can induce progressive neurological deficit presenting with infarct enlargement [3]. Arterial stiffness is a risk factor for progressive neurological deficit [3]. The major mechanism of lacunar infarcts is thought to involve traditional risk factors such as hypertension and diabetes mellitus [22], although cerebral SVD associated with arterial stiffness may also be an important contributor.

There are some clinical differences between the various surrogate markers of arterial stiffness used in patients with ischemic stroke: (1) a low ABI indicates advanced atherosclerosis, (2) an increased PWV strongly indicates the presence of cerebral SVD, and (3) an increased CAVI may indicate vessel stiffness due to arteriosclerosis [18]. Cerebral SVD may be associated with any of these surrogate markers. Such clinical differences and associations should be clarified in detail in future studies.

\section{Cognitive Impairment}

Cerebral SVD such as SLI,WMH, and MB are well-known risk factors for cognitive impairment $[1,2]$. Cognitive impairment including dementia is also associated with arterial stiffness [8-12]. An $A B I>1.30$ and increased blood pressure variability are important predictors of cognitive impairment among patients without vascular diseases [13]. Thus, an increased ABI indicates arterial calcification that leads to vessel sclerosis (advanced vessel stiffness). Increased PWV is also associated with cognitive impairment $[12,14]$, an independent predictor of cognitive decline $[10,11]$, and $\beta$-amyloid $(A \beta)$ deposition in the brains of older patients [15]. More specifically, Hughes et al. [15] showed the associations between arterial stiffness and $A \beta$ deposition in the brain independent of blood pressure. Increased arterial stiffness has a direct impact on penetrating arteries of the brain, leading to an altered structure and function, with subsequent effects on perivascular amyloid clearance from the brain via the cerebrospinal fluid drainage along the perivascular space. This may contribute to a disruption of vascular dynamics and complicate perivascular flow of $A \beta$, thus indirectly causing decreased $A \beta$ clearance leading to plaque formation. Therefore, arterial stiffness may contribute to the pathogenesis of cognitive impairment through its potential role in the effects of hypertension on the brain, cerebrovascular disease, vascular dementia, and Alzheimer's disease pathology [24].

There are two vessel-related mechanisms thought to be involved in cognitive impairment: cerebral amyloid angiopathy and hypertensive SVD. The etiology of MB is associated with both cerebral amyloid angiopathy and hypertensive SVD [29], and both may be associated with arterial stiffness. Therefore, MB are a surrogate marker of both vascular cognitive impairment and stroke. Collectively, the risk of cognitive impairment depends on the presence of any components of cerebral SVD including WMH, SLI, and MB, all of which are associated with arterial stiffness. 
Saji et al.: Cerebral Small Vessel Disease and Arterial Stiffness: Tsunami Effect in the Brain?

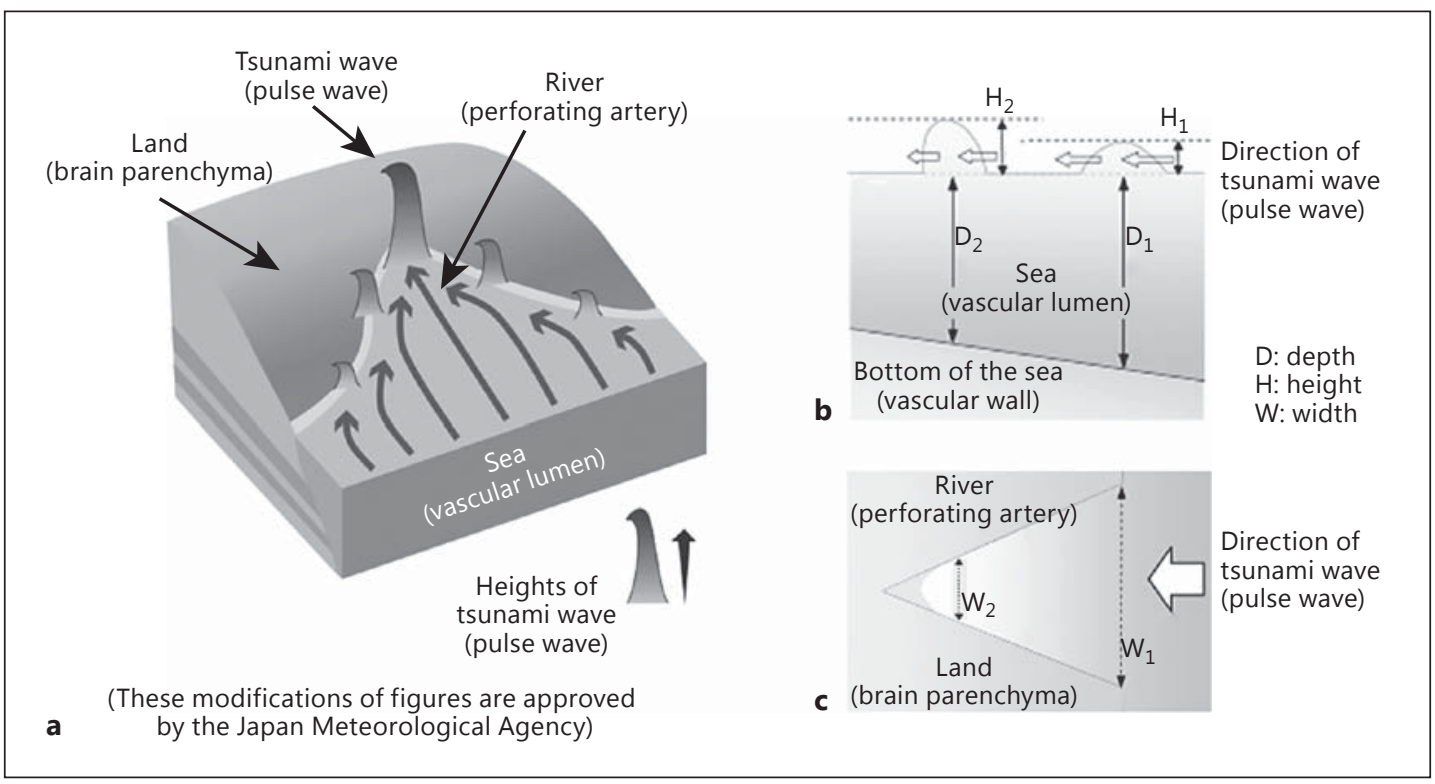

Fig. 1. Overview of the tsunami wave model. a The heights of tsunami waves increase as the waves move closer to the land because of the geographical features of the bottom of the sea and/or river and those of the seashore. This mechanism is due to the depths (b) and the widths (c) of the bay and/or river. Words in parentheses are added by the authors to suggest how this tsunami wave model may explain the mechanism of cerebral damage in cerebral SVD, cognitive impairment, and the geriatric syndrome due to increased PWV. This figure is cited from [33]. b Vertical section of the wave. The heights of the tsunami waves increase $\left(\mathrm{H}_{1} \rightarrow \mathrm{H}_{2}\right)$ as the waves move closer to the land because the depths of the sea and/or river $\left(\mathrm{D}_{1} \rightarrow \mathrm{D}_{2}\right)$ become shallower. c Horizontal section of the wave. The heights of tsunami waves increase $\left(\mathrm{H}_{1} \rightarrow \mathrm{H}_{2}\right)$ as the waves move closer to the land because the widths of the sea and/or river $\left(\mathrm{W}_{1} \rightarrow \mathrm{W}_{2}\right)$ become narrower.

\section{Geriatric Syndrome}

The geriatric syndrome is defined as having clinical symptoms characteristic of older adults, typically including cognitive functional impairment, falls, foreign body aspiration, impaired appetite of unknown cause, frailty, and sarcopenia [30]. This syndrome can be associated with cerebral SVD, as shown above. Recently, novel associations between the geriatric syndrome and arterial stiffness have been reported. Frailty syndrome in older adults is associated with subclinical peripheral arterial disease indicated by ABI [4]. Increased brachialankle PWV is also associated with an increased risk for mortality and the onset of impairment in activities of daily living [5, 7]. These factors may pose risks for dementia; this is an issue that should be investigated in more detail in the future.

\section{Mechanism}

Numerous studies have reported associations between arterial stiffness and various patterns of cerebral damage, as well as injury to other organs such as the retina, heart, and kidney. These organs have a common vascular mechanism of injury consisting of perforating arteries. This mechanism has been indicated as a water hammer effect [31]. In detail, Fazekas et al. [31] suggested the pathological findings of WMH as follows: arteries with walls thickened by hyalinosis and fibrosis, large perivascular spaces bordered by atrophic neuropil, and extensive arterial pulsations which could be implicated in their pathogenesis by initiating a 
Fig. 2. Schema of the associations between factors related to arterial stiffness. HT = Hypertension; $\mathrm{DM}=$ diabetes mellitus; DL = dyslipidemia; $\mathrm{AI}=$ augmentation index. This figure is cited from [33].

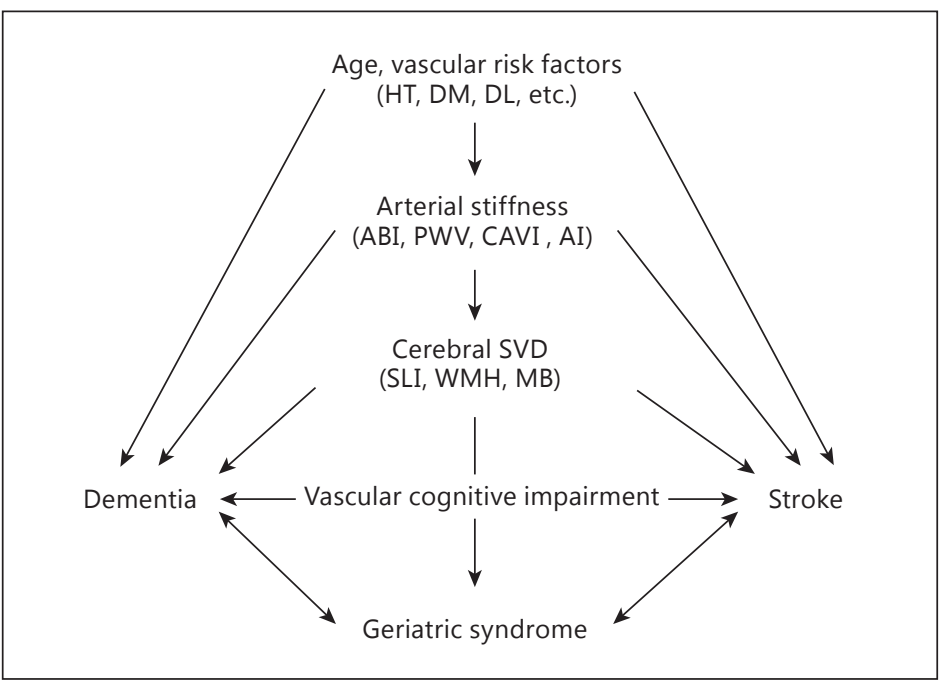

water hammer effect on the surrounding tissue. Recently, some researchers have suggested physiological considerations [27, 32]. Van Sloten et al. [27] showed that increased pulsatile pressure and flow might directly load to cerebral microcirculatory damage and induce a microvascular remodeling response. O'Rourke and Hashimoto [32] also showed in detail that repetitive pulsations cause fatigue and fracture of elastin lamellae of central arteries, causing them to stiffen, so that reflections return earlier to the heart; in consequence, aortic systolic pressure rises, diastolic pressure falls, and pulsations of flow extend further into cerebral small arteries, which leads to damage to the cerebral parenchyma. Stiffening also increases stresses on cerebral small arteries.

Based on our findings and previous studies, we propose a hypothesis using the 'tsunami wave model' to improve our understanding of the risk and the importance of arterial stiffness (fig. 1) [33]. The tsunami wave model shows that wave damage to the land increases with river narrowing (fig. 1a). The mechanism involves an increase in tsunami wave height as the wave moves closer to the land, owing to the geographical features of the bottom of the sea and/or the width of the river (fig. 1b, c). The power of the tsunami wave depends on the depth and width of the bay and/or river. This tsunami wave model could explain the physiological action of increased arterial stiffness. Although both a water hammer model and this tsunami wave model might indicate a similar physiological mechanism [32], this mechanism could also contribute to an improved understanding of the association between cerebral SVD and increased arterial stiffness.

Vascular narrowing due to atherosclerosis and vascular stiffness due to lipohyalinosis may accelerate the pulse waves. Based on this mechanism, we also propose a hypothesis regarding the connection of vascular risk factors, cerebral SVD, and stroke with cognitive impairment via arterial stiffness (fig. 2) [33]. This hypothesis will be investigated in more detail to clarify the mechanism in future studies.

\section{Conclusion}

Cerebral SVD is a well-known and important visual finding on brain MRI in older patients with and without risk factors for cerebrovascular diseases and cognitive impairment. The vascular, physiological, and metabolic roles of arterial stiffness require detailed clarification 
in future studies. Further, improvement of the living environment, management of risk factors, and innovation and development of novel drugs may help decrease the progression of cerebral SVD and reduce the risk of stroke and dementia.

\section{Acknowledgments}

We thank Kyoko Banno and Natsuyo Kimoto for secretarial assistance.

\section{Disclosure Statement}

This work was financially supported by grants from the Research Funding of Longevity Sciences (25-6, 27-21) from the National Center for Geriatrics and Gerontology and by a research grant (Grants-in-Aid for Scientific Research, No. 26870765) from the Japan Society for the Promotion of Science.

\section{References}

1 Wardlaw JM, Smith EE, Biessels GJ, et al; Standards for Reporting Vascular Changes on Neuroimaging (STRIVE v1): Neuroimaging standards for research into small vessel disease and its contribution to ageing and neurodegeneration. Lancet Neurol 2013;12:822-838.

2 Pantoni L: Cerebral small vessel disease: from pathogenesis and clinical characteristics to therapeutic challenges. Lancet Neurol 2010;9:689-701.

3 Saji N, Kimura K, Kawarai T, Shimizu H, Kita Y: Arterial stiffness and progressive neurological deficit in patients with acute deep subcortical infarction. Stroke 2012;43:3088-3090.

4 Lin CH, Chou CY, Liu CS, Huang CY, Li TC, Lin CC: Association between frailty and subclinical peripheral vascular disease in a community-dwelling geriatric population: Taichung Community Health Study for Elders. Geriatr Gerontol Int 2015;15:261-267.

5 Kuroiwa Y, Miyano I, Nishinaga M, Takata J, Shimizu Y, Okumiya K, Matsubayashi K, Ozawa T, Kitaoka H, Doi Y, Yasuda N: Association between level of brachial-ankle pulse wave velocity and onset of activities of daily living impairment in community-dwelling older individuals. Geriatr Gerontol Int 2015;15:840-847.

6 Saji N, Kimura K, Ohsaka G, Higashi Y, Teramoto Y, Usui M, Kita Y: Functional independence measure scores predict level of long-term care required by patients after stroke: a multicenter retrospective cohort study. Disabil Rehabil 2015;37:331-337.

7 Takashima N, Turin TC, Matsui K, Rumana N, Nakamura Y, Kadota A, Saito Y, Sugihara H, Morita Y, Ichikawa M, Hirose K, Kawakani K, Hamajima N, Miura K, Ueshima H, Kita Y: The relationship of brachial-ankle pulse wave velocity to future cardiovascular disease events in the general Japanese population: the Takashima Study. J Hum Hypertens 2014;28:323-327.

8 Kim J, Song TJ, Song D, Lee KJ, Kim EH, Lee HS, Nam CM, Nam HS, Kim YD, Heo JH: Brachial-ankle pulse wave velocity is a strong predictor for mortality in patients with acute stroke. Hypertension 2014;64:240-246.

9 Gronewold J, Hermann DM, Lehmann N, Kröger K, Lauterbach K, Berger K, Weimar C, Kälsch HIM, Moebus S, Jöckel KH, Bauer M, Erbel R; Heinz Nixdorf Recall Study Investigative Group: Ankle-brachial index predicts stroke in the general population in addition to classical risk factors. Atherosclerosis 2014;233:545-550.

10 Yukutake T, Yamada M, Fukutani N, Nishiguchi S, Kayama H, Tanigawa T, Adachi D, Hotta T, Morino S, Tashiro Y, Aoyama T, Arai H: Arterial stiffness predicts cognitive decline in Japanese community-dwelling elderly subjects: a one-year follow-up study. J Atheroscler Thromb 2015;22:637-644.

11 Taniguchi Y, Fujiwara Y, Nofuji Y, Nishi M, Murayama H, Seino S, Tajima R, Matsuyama Y, Shinkai S: Prospective study of arterial stiffness and subsequent cognitive decline among community-dwelling older Japanese. J Epidemiol 2015;25:592-599.

12 Scuteri A, Wang H: Pulse wave velocity as a marker of cognitive impairment in the elderly. J Alzheimers Dis 2014;42(suppl 4):S401-S410.

13 Gutierrez J, Marshall RS, Lazar RM: Indirect measures of arterial stiffness and cognitive performance in individuals without traditional vascular risk factors or disease. JAMA Neurol 2015;72:309-315.

14 Zhong W, Cruickshanks KJ, Schubert CR, Carlsson CM, Chappell RJ, Klein BE, Klein R, Acher CW: Pulse wave velocity and cognitive function in older adults. Alzheimer Dis Assoc Disord 2014;28:44-49.

15 Hughes TM, Kuller LH, Barinas-Mitchell EJ, Mackey RH, McDade EM, Klunk WE, Aizenstein HJ, Cohen AD, Snitz BE, Mathis CA, Dekosky ST, Lopez OL: Pulse wave velocity is associated with $\beta$-amyloid deposition in the brains of very elderly adults. Neurology 2013;81:1711-1718. 
Saji et al.: Cerebral Small Vessel Disease and Arterial Stiffness: Tsunami Effect in the Brain?

16 Yamashina A, Tomiyama H, Takeda K, Tsuda H, Arai T, Hirose K, Koji Y, Hori S, Yamamoto Y: Validity, reproducibility, and clinical significance of noninvasive brachial-ankle pulse wave velocity measurement. Hypertens Res 2002;25:359-364.

17 Shirai K, Hiruta N, Song M, Kurosu T, Suzuki J, Tomaru T, Miyashita Y, Saiki A, Takahashi M, Suzuki K, Takata M: Cardia-ankle vascular index (CAVI) as a novel indicator of arterial stiffness: theory, evidence and perspectives. J Atheroscler Thromb 2011;18:924-938.

18 Saji N, Kimura K, Yagita Y, Kawarai T, Shimizu H, Kita Y: Comparison of arteriosclerotic indicators in patients with ischemic stroke: ankle-brachial index, brachial-ankle pulse wave velocity, and cardio-ankle vascular index. Hypertens Res 2015;38:323-328.

19 Saji N, Shimizu H, Kawarai T, Tadano M, Kita Y, Yokono K: Increased brachial-ankle pulse wave velocity is independently associated with white matter hyperintensities. Neuroepidemiology 2011;36:252-257.

20 Saji N, Kimura K, Shimizu H, Kita Y: Silent brain infarct is independently associated with arterial stiffness indicated by cardio-ankle vascular index (CAVI). Hypertens Res 2012;35:756-760.

21 Saji N, Kimura K, Yagita Y, Uemura J, Aoki J, Sato T, Sakurai T: Deep cerebral microbleeds and chronic kidney disease in patients with acute lacunar infarcts. J Stroke Cerebrovasc Dis 2015;11:2572-2579.

22 Saji N, Shimizu H, Kawarai T, Tadano M, Kita Y, Yokono K: Clinical features of a first-ever lacunar infarction in Japanese patients: poor outcome in females. J Stroke Cerebrovasc Dis 2011;20:231-235.

23 Lau KK, Wong YK, Chan YH, Teo KC, Chan KH, Wai Li LS, Cheung RT, Siu CW, Ho SL, Tse HF: Visit-to-visit blood pressure variability as a prognostic marker in patients with cardiovascular and cerebrovascular diseases: relationships and comparisons with vascular markers of atherosclerosis. Atherosclerosis 2014;235:230-235.

24 Tzourio C, Laurent S, Debette S: Is hypertension associated with an accelerated aging of the brain? Hypertension 2014;63:894-890.

25 Saji N, Kimura K, Shimizu H, Kita Y: Association between silent brain infarct and arterial stiffness indicated by brachial-ankle pulse wave velocity. Intern Med 2012;51:1003-1008.

26 Seo WK, Lee JM, Park MH, Park KW, Lee DH: Cerebral microbleeds are independently associated with arterial stiffness in stroke patients. Cerebrovasc Dis 2008;26:618-623.

27 van Sloten TT, Protogerou AD, Henry RM, Schram MT, Launer LJ, Stehouwer CD: Association between arterial stiffness, cerebral small vessel disease and cognitive impairment: a systematic review and meta-analysis. Neurosci Biobehav Rev 2015;53:121-130.

28 Saji N, Sato T, Sakuta K, Aoki J, Kobayashi K, Matsumoto N, Uemura J, Shibazaki K, Kimura K: Chronic kidney disease is an independent predictor of adverse clinical outcomes in patients with recent small subcortical infarcts. Cerebrovasc Dis Extra 2014;4:174-181.

29 Tomimoto H: Subcortical vascular dementia. Neurosci Res 2011;71:193-199.

30 Arai H, Ouchi Y, Toba K, Endo T, Shimokado K, Tsubota K, Matsuo S, Mori H, Yumura W, Yokode M, Rakugi H, Ohshima S: Japan as the front-runner of super-aged societies: perspectives from medicine and medical care in Japan. Geriatr Gerontol Int 2015;15:673-687.

31 Fazekas F, Kleinert R, Offenbacher H, Payer F, Schmidt R, Kleinert G, Radner H, Lechner H: The morphologic correlate of incidental punctate white matter hyperintensities on MR images. AJNR Am J Neuroradiol 1991; 12:915-921.

32 O'Rourke MF, Hashimoto J: Mechanical factors in arterial aging: a clinical perspective. J Am Coll Cardiol 2007; 50:1-13.

33 Saji N, Ogama N, Toba K, Sakurai T: White matter hyperintensities and the geriatric syndrome: an important role of arterial stiffness. Geriatr Gerontol lnt 2015;15(suppl 1):17-25. 